\title{
DESENVOLVIMENTO E PRODUÇÃO DE FRUTOS DE BANANEIRA EM RESPOSTA À ADUBAÇÃO NITROGENADA E POTÁSSICA ${ }^{1}$
}

\author{
EDILSON CARVALHO BRASIL ${ }^{2}$, ANTÔNIO HENRIQUE LOPES OEIRAS ${ }^{3}$, \\ ANTÔNIO JOSÉ ELIAS AMORIM DE MENEZES ${ }^{4}$ e CARLOS ALBERTO COSTA VELOSO 5
}

\begin{abstract}
RESUMO - Avaliou-se o efeito da adubação nitrogenada e potássica no desenvolvimento e produção da bananeira (Musa spp.), cultivar Pioneira, em experimento conduzido no Município de Capitão Poço, PA, em Latossolo Amarelo, utilizando-se o delineamento experimental em blocos casualizados. Os tratamentos foram: $0,80,160$ e $240 \mathrm{~g}$ de N/planta/ano e 0, 150, 300 e $450 \mathrm{~g}$ de $\mathrm{K}_{2} \mathrm{O} /$ planta/ano. Como fontes de nutrientes, utilizaram-se uréia e cloreto de potássio. Os resultados de crescimento, até 240 dias do plantio, indicaram que apenas o $\mathrm{N}$ influenciou a circunferência do pseudocaule e a altura de planta, verificando-se que o modelo quadrático ajustou-se melhor a todas as variáveis avaliadas. No segundo ciclo de produção, a adição de $\mathrm{K}$ promoveu efeito quadrático no peso de cacho, peso de penca por cacho e peso médio de penca, com incrementos de 73,76 e 39\%, respectivamente, em relação à ausência de $\mathrm{K}$. A aplicação de $\mathrm{N}$ promoveu aumento linear no peso de cacho e de pencas por cacho, com aumentos máximos de 32 e $30 \%$, respectivamente, em relação a ausência do nutriente. No terceiro ciclo de produção, apenas o K influenciou no peso de cacho, peso de penca por cacho e peso médio de penca, com aumentos de 39,40 e $26 \%$, respectivamente.
\end{abstract}

Termos para indexação: Musa, nutrientes minerais, absorção de nutrientes, produtividade.

\section{DEVELOPMENT AND PRODUCTION OF FRUITS OF BANANA PLANT AS A RESPONSE TO NITROGEN AND POTASSIUM FERTILIZATION}

\begin{abstract}
The effect of the $\mathrm{N}$ and $\mathrm{K}$ fertilization on the development and production of the banana plant (Musa spp.), cv. Pioneira, was evaluated in an experiment carried out in Capitão Poço county, Pará State, Brazil, in a Yellow Latosol, using a randomized blocks design, in factorial arrangement of $4 \mathrm{x} 4$. The treatments were: $0,80,160$ and $240 \mathrm{~g}$ of $\mathrm{N}$ per plant per year; $0,150,300$ and $450 \mathrm{~g}$ of $\mathrm{K}_{2} \mathrm{O}$ per plant per year. Urea and potassium chloride were used as nutrient sources. The results of plant growth showed that there was effect of $\mathrm{N}$ on circumference of pseudostem and plant height. The quadratic model was better adjusted to all the variables. The results of production of the second cycle indicated that $\mathrm{K}$ increased quadratically the bunch weight, hands weight per bunch and average weight of hands, with production increments of 73, 76 and 39\%, respectively, in comparison to the treatment without potassium. The nitrogen application promoted a linear increase of the weight of bunch and hands per bunch, with increments of 32 and $30 \%$, respectively, proportionate by the highest level of $\mathrm{N}$, in relation to the treatment without this nutrient. During the third production cycle only $\mathrm{K}$ application influenced the weights of bunch, of hands per bunch and average weight of hands, with increments of $39,40 \%$ and $26 \%$, respectively.
\end{abstract}

Index terms: Musa, mineral nutrients, nutrient uptake, productivity.

\footnotetext{
${ }^{1}$ Aceito para publicação em 21 de janeiro de 2000.

Trabalho realizado com apoio financeiro da Empresa Cítricos do Pará S.A. (CITROPAR).

${ }^{2}$ Eng. Agrôn., M.Sc., Embrapa-Centro de Pesquisa Agroflorestal da Amazônia Oriental (CPATU), Caixa Postal 48, CEP 66095-100 Belém, PA. E-mail: brasil@cpatu.embrapa.br
}

\footnotetext{
${ }^{3}$ Eng. Agrôn., Citropar, Rod. PA-253, km 4, CEP 68650-000

Capitão Poço, PA. E-mail: citricos@zaz.com.br

${ }^{4}$ Eng. Agrôn., Embrapa-CPATU.

E-mail: toninho@cpatu.embrapa.br

${ }^{5}$ Eng. Agrôn., D.Sc., Embrapa-CPATU.

E-mail: veloso@cpatu.embrapa.br
} 


\section{INTRODUÇÃO}

A bananeira é uma das fruteiras mais exploradas nos países tropicais e seu fruto um dos mais consumidos no mundo. Há vários anos o Brasil destaca-se como um dos principais produtores mundiais, com cultivo da fruteira na maioria dos estados da Federação.

Do total de frutos produzidos no país, apenas $1 \%$ é exportado. O restante da produção é comercializado no mercado interno, indicando que o fruto representa um importante complemento na dieta alimentar da população de baixa renda.

No Estado do Pará, a bananeira é uma das culturas com maior expansão em área de cultivo, estando entre as de principal interesse econômico (Coral, 1998). Atualmente, em termos de produção de frutos, o Pará apresenta posição de destaque no cenário nacional. No entanto, a maioria das áreas cultivadas encontra-se implantada em solos considerados pobres em nutrientes. A utilização de solos pouco férteis e a falta de manutenção de níveis adequados de nutrientes durante o ciclo da planta são os principais fatores da baixa produtividade da bananeira (Borges \& Oliveira, 1995). Aliados a isso, as elevadas quantidades de nutrientes retirados do solo durante os vários ciclos produtivos da cultura (Borges et al., 1995) podem comprometer ainda mais o equilíbrio nutricional da planta.

A extração de nutrientes do período juvenil até o lançamento do cacho é bastante acentuada, quando são verificadas grandes retiradas de $\mathrm{K}, \mathrm{N}, \mathrm{Ca}, \mathrm{P}$ e Mg (Martin-Prével, 1964; Gallo et al., 1972). O K e o N estão diretamente relacionados com o crescimento, produção e qualidade dos frutos da bananeira.

O K é o macronutriente extraído em maiores quantidades pela planta, por causa da ação direta nas trocas metabólicas, no transporte da seiva elaborada, na retenção de água e nas qualidades organolépticas do fruto (Martin-Prével \& Montagut, 1966). A assimilação deste nutriente está inteiramente ligada à do N, havendo uma relação específica entre eles, que varia de acordo com diversos tipos de solo, clima e cultivar (Moreira, 1987).

Por sua vez, o N é muito importante para a bananeira, atuando diretamente sobre o crescimento vegetativo, emissão e desenvolvimento dos reben- tos, além de aumentar a quantidade de matéria seca (Borges et al., 1995).

Como forma de aumentar a expressão produtiva e atender às exigências nutricionais da cultura, a prática da adubação requer o fornecimento de nutrientes na forma adequada e na quantidade desejada.

Apesar de a bananeira ser cultivada há bastante tempo no Pará, poucas são as informações disponíveis baseadas em resultados experimentais sobre recomendações de adubação para a cultura nas condições do Estado.

O objetivo do trabalho foi avaliar o efeito da aplicação de doses de N e K no desenvolvimento e produção da bananeira, cultivar Pioneira, nas condições de solo e clima do Pará.

\section{MATERIAL E MÉTODOS}

O experimento foi conduzido na Fazenda da Empresa Cítricos do Pará SA - CITROPAR, no Município de Capitão Poço, PA, em um Latossolo Amarelo, textura média, com as seguintes características químicas (Embrapa, 1979), na profundidade de $0-20 \mathrm{~cm}$ : pH (água), 5,4; MO, 13,8 $\mathrm{g} \mathrm{dm}^{-3}$; P, $2 \mathrm{mg} \mathrm{kg}^{-1}$; K, 0,5 $\mathrm{mmol}_{\mathrm{c}} \mathrm{dm}^{-3}$; $\mathrm{Ca}+\mathrm{Mg}, 7 \mathrm{mmol}_{\mathrm{c}} \mathrm{dm}^{-3}$; e $\mathrm{Al}, 1 \mathrm{mmol}_{\mathrm{c}} \mathrm{dm}^{-3}$.

Utilizou-se o delineamento experimental em blocos casualizados, com três repetições, em arranjo fatorial $4 \times 4$, correspondendo a quatro níveis de $\mathrm{N}$ e quatro níveis de $\mathrm{K}$.

Os tratamentos corresponderam à combinação dos níveis dos fatores, empregando-se as seguintes doses: 0,80 , 160 e $240 \mathrm{~g}$ de N/planta/ano; 0, 150, 300 e $450 \mathrm{~g}$ de $\mathrm{K}_{2} \mathrm{O} /$ planta/ano. Como fontes de nutrientes foram utilizados uréia e cloreto de potássio, aplicados em cobertura, em semicírculo, na projeção da copa. As adubações foram realizadas, parceladamente, em quatro vezes, com a primeira aplicação aos 35 dias após o plantio e as restantes de 40 em 40 dias.

Em todas as parcelas efetuou-se uma adubação básica na cova de plantio com cinco litros de esterco de carneiro, $190 \mathrm{~g}$ de $\mathrm{P}_{2} \mathrm{O}_{5}$, na forma de superfosfato triplo e $300 \mathrm{~g}$ de calcário dolomítico.

As parcelas foram constituídas de 20 plantas na área total e seis plantas na área útil. O plantio foi efetuado em março de 1996, no início do período chuvoso, em covas de $0,4 \mathrm{~m} \times 0,4 \mathrm{~m} \times 0,4 \mathrm{~m}$, no espaçamento de $3 \mathrm{~m} \times 2 \mathrm{~m}$, utilizando-se mudas de bananeira, cultivar Pioneira, do tipo chifre e chifrinho.

Para avaliação do desenvolvimento das plantas, foram realizadas medições biométricas, aos 70, 140 e 240 dias após o plantio, da circunferência do pseudocaule a $30 \mathrm{~cm}$ 
do nível do solo e altura da planta. Foram calculados incrementos de altura, efetuando-se a subtração dos valores obtidos entre a segunda e primeira medição de altura e dos valores obtidos entre a última e primeira.

As variáveis de produção foram obtidas somente do segundo (filha) e terceiro (neta) ciclos, desconsiderando-se a produção do primeiro ciclo (mãe), em virtude da desuniformidade das plantas. Para fins de análise estatística, coletaram-se dados de peso de cacho, peso de penca por cacho, peso médio de penca, número de banana por cacho e número de penca por cacho.

Os resultados foram avaliados estatisticamente mediante análise de variância (teste F) em relação a todas as variáveis obtidas. Para avaliação dos níveis de $\mathrm{N}$ e $\mathrm{K}$ foram ajustadas curvas de regressão, seguindo o modelo polinomial que melhor ajustou-se ao conjunto de dados.

\section{RESULTADOS E DISCUSSÃO}

A análise de variância dos dados de desenvolvimento da bananeira revelou que somente houve efeito significativo de doses de $\mathrm{N}$, a $5 \%$ de probabilidade, para circunferência de caule obtida aos 140 e 240 dias, para altura de planta medida aos 70, $140 \mathrm{e}$ 240 dias e para incremento de altura obtido entre a primeira e a terceira mensuração (Tabela 1). Os tratamentos com doses de K não apresentaram efeito significativo em relação às variáveis estudadas. A análise de regressão para o efeito de doses de $\mathrm{N}$ indicou que o modelo quadrático foi o que melhor explicou os resultados obtidos, para todas as variáveis de resposta (Fig. 1).

Considerando-se a estimativa de desenvolvimento ótimo da bananeira (90\% da máxima resposta da planta), a análise de regressão permitiu estabelecer que as melhores respostas, em termos de desenvolvimento inicial, foram obtidas com a aplicação de 57; 44 e $13 \mathrm{~g}$ de N por planta, respectivamente, para altura de planta nas avaliações realizadas aos 70, 140 e 240 dias (Fig. 1a). As melhores respostas em circunferência de pseudocaule foram conseguidas com a adição de 56 e $3 \mathrm{~g}$ de $\mathrm{N}$ por planta, respectivamente, aos 140 e 240 dias (Fig. 1b). Tais resultados indicam que a cultura exigiu maiores quantidades de $\mathrm{N}$ nos primeiros meses de desenvolvimento, concordando com Borges et al. (1995) que consideram o nutriente muito importante para o crescimento vegetativo da bananeira. Para Warner \& Fox (1977) o N tem grande influência no crescimento inicial da bananeira, principalmente quando o meristema encontra-se em fase de desenvolvimento. Nas avaliações realizadas após o quarto mês do plantio (aos 240 dias), observou-se que as quantidades de $\mathrm{N}$ exigidas para obtenção do desenvolvimento ótimo foram bastante reduzidas, em relação às avaliações anteriores.

Diversos autores (Hewitt, 1955; Twyford \& Walmsley, 1974; Neves et al., 1991) mencionaram que a bananeira, independentemente da cultivar, extrai maiores quantidades de $\mathrm{K}$ que de $\mathrm{N}$, durante o ciclo de formação da planta. Contudo, esse aspecto não se contrapõe aos resultados obtidos, pois a não ocorrência de efeito para a aplicação de K indica que a planta utilizou-se das reservas do solo para suprir as exigências nutricionais do elemento, durante essa fase.

A análise de variância dos dados do segundo ciclo de produção da bananeira, cv. Pioneira, indicou

TABELA 1. Valores de $\mathrm{F}$ da análise de variância em relação às variáveis de crescimento da bananeira, cv. Pioneira, aos 70, 140 e 240 dias após o plantio.

\begin{tabular}{lcccccccc}
\hline \multirow{2}{*}{$\begin{array}{c}\text { Fontes de } \\
\text { variação }\end{array}$} & DM1 & DM2 & DM3 & ALT1 & ALT2 & ALT3 & INCRALT1 & INCRALT2 \\
\cline { 2 - 8 } $\mathrm{N}$ & $2,47^{\text {ns }}$ & $3,80^{*}$ & $4,03^{*}$ & $5,51^{*}$ & $3,68^{*}$ & $3,97^{*}$ & $2,23^{\text {ns }}$ & $3,30^{*}$ \\
$\mathrm{~K}$ & $1,27^{\text {ns }}$ & $1,54^{\text {ns }}$ & $2,74^{\text {ns }}$ & $1,67^{\text {ns }}$ & $2,19^{\text {ns }}$ & $2,60^{\text {ns }}$ & $1,92^{\text {ns }}$ & $2,85^{\text {ns }}$ \\
NxK & $0,65^{\text {ns }}$ & $1,19^{\text {ns }}$ & $0,78^{\text {ns }}$ & $0,97^{\text {ns }}$ & $1,24^{\text {ns }}$ & $1,15^{\text {ns }}$ & $1,09^{\text {ns }}$ & $0,96^{\text {ns }}$ \\
\hline CV $(\%)$ & 21,90 & 25,02 & 14,24 & 18,97 & 20,16 & 12,83 & 28,52 & 16,58 \\
Média & 4,52 & 10,23 & 14,17 & 38,96 & 95,75 & 121,00 & 56,94 & 82,25 \\
\hline
\end{tabular}

1 DM1, DM2 e DM3: circunferência do pseudocaule aos 70, 140 e 240 dias após o plantio (cm); ALT1, ALT2 e ALT3: altura de planta aos 70, 140 e 240 dias após o plantio $(\mathrm{cm})$; INCRALT1: incremento entre a primeira e segunda medições de altura $(\mathrm{cm})$; INCRALT2: incremento entre a primeira e terceira medições de altura $(\mathrm{cm})$.

ns e * Não-significativo e significativo a $5 \%$ de probabilidade, respectivamente. 
que houve efeito significativo em todas as variáveis estudadas, em função da aplicação de doses de K, como pode ser verificado na Tabela 2. Observou-se, também, resposta de doses de $\mathrm{N}$ na maioria das variáveis, com exceção de peso médio de penca.

De acordo com os resultados obtidos, a adição de doses de $\mathrm{K}$ proporcionou aumentos de forma
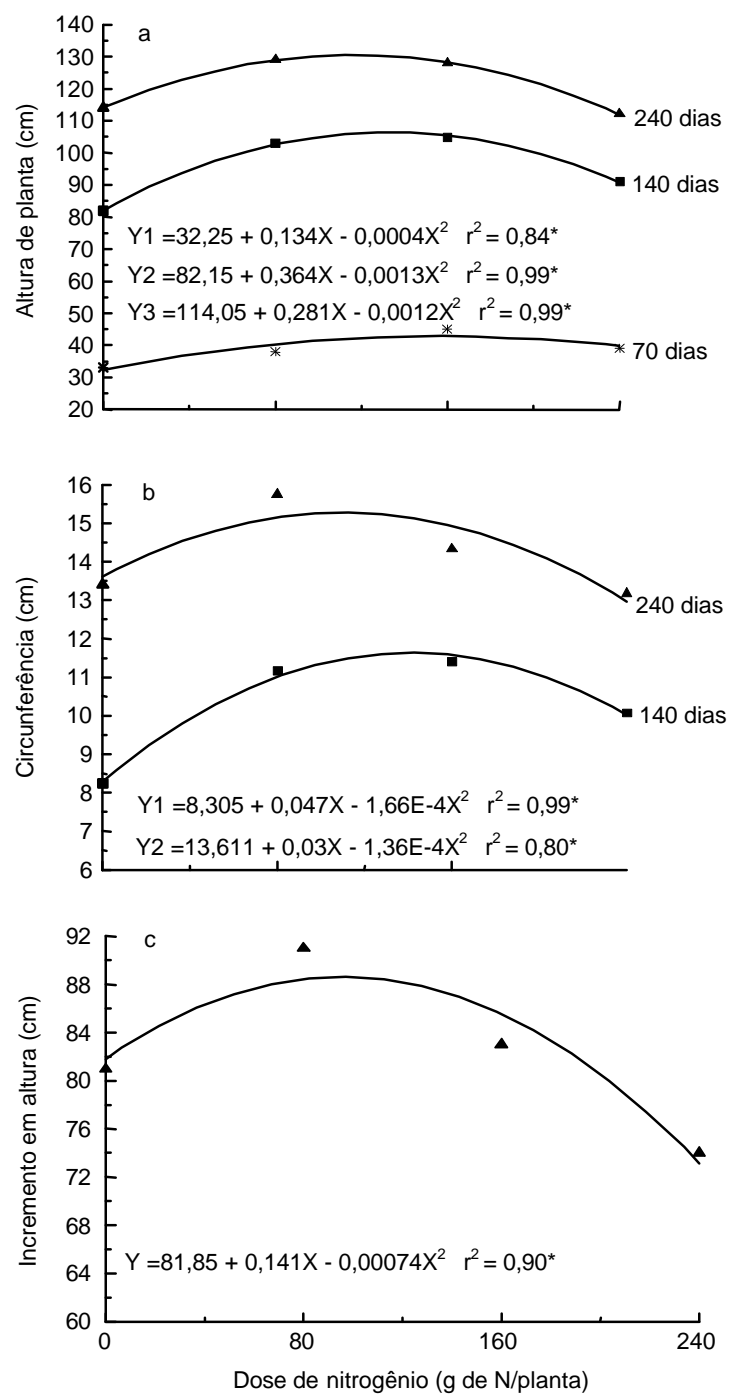

FIG. 1. Efeito de doses de $\mathbf{N}$ sobre a altura de planta (a), circunferência do pseudocaule (b) $e$ incremento de altura de planta entre a primeira e terceira medição (c) de bananeira, cv. Pioneira. quadrática em relação a peso de cacho, peso de penca por cacho e peso médio de penca (Fig. 2), enquanto nas variáveis número de penca por cacho e número de banana por cacho observou-se resposta linear (Fig. 3).

Considerando-se a estimativa de produção ótima, verificou-se que, para a obtenção de $90 \%$ da máxima produção física, seriam necessárias aplicações de 222,221 e $167 \mathrm{~g}$ de $\mathrm{K}_{2} \mathrm{O}$ por planta, respectivamente, com respeito a peso de cacho, peso de penca por cacho e peso médio de penca. Tomando-se por base esses valores, observaram-se boas respostas em termos de produção da bananeira, verificando-se incrementos da ordem de $73 \%, 76 \%$ e $39 \%$, respectivamente, nessas mesmas variáveis, em relação à ausência de K. Manica et al. (1978) também obtiveram resultados positivos com a aplicação de $180 \mathrm{~g} /$ cova/ano de $\mathrm{K}_{2} \mathrm{O}$, conseguindo aumentos de produção de 37\%. Cunha \& Fraga Júnior (1963) verificaram que, em bananais adultos de 10-18 anos de idade, somente a adição de doses iniciais de 190 e $380 \mathrm{~g} /$ planta de $\mathrm{K}_{2} \mathrm{O}$, foi suficiente para promover aumentos de $133 \%$ e $53 \%$ no rendimento de frutos, respectivamente, em duas localidades estudadas.

TABELA 2. Valores de $F$ da análise de variância em relação às variáveis de produção da bananeira, cv. Pioneira, obtidos no segundo e terceiro ciclos de produção.

\begin{tabular}{lccccc}
\hline \multirow{2}{*}{$\begin{array}{c}\text { Fontes de } \\
\text { variação }\end{array}$} & PC & PP & NPC & PMP & NBC \\
\cline { 2 - 6 } & \multicolumn{5}{c}{$2^{\text {o ciclo produtivo }}-1997$} \\
BL & 1,16 & 1,54 & 2,92 & 0,68 & 3,78 \\
N & $3,76^{*}$ & $3,54^{*}$ & $3,44^{*}$ & $2,01^{\text {ns }}$ & $3,27^{*}$ \\
$\mathrm{~K}$ & $16,40^{*}$ & $16,69^{*}$ & $10,25^{*}$ & $13,37^{*}$ & $8,99^{*}$ \\
NxK & $1,94^{\text {ns }}$ & $2,04^{\text {ns }}$ & $0,86^{\text {ns }}$ & $2,22^{\text {ns }}$ & $0,90^{\text {ns }}$ \\
\hline CV $(\%)$ & 21,90 & 22,30 & 10,70 & 17,10 & 15,60 \\
Média & 5,35 & 4,81 & 5,57 & 0,84 & 61,19 \\
\hline \multicolumn{5}{c}{$3^{\circ}$ ciclo produtivo -1998} \\
BL & 6,35 & 7,20 & 8,33 & 2,64 & 4,86 \\
N & $0,87^{\text {ns }}$ & $1,38^{\text {ns }}$ & $1,83^{\text {ns }}$ & $0,71^{\text {ns }}$ & $1,15^{\text {ns }}$ \\
K & $5,50^{*}$ & $5,29 *$ & $1,75^{\text {ns }}$ & $5,85^{*}$ & $1,48^{\text {ns }}$ \\
NxK & $0,62^{\text {ns }}$ & $0,66^{\text {ns }}$ & $1,37^{\text {ns }}$ & $0,72^{\text {ns }}$ & $1,09^{\text {ns }}$ \\
\hline CV $(\%)$ & 25,50 & 26,50 & 18,20 & 19,60 & 25,00 \\
Média & 5,55 & 4,86 & 6,73 & 0,73 & 72,10 \\
\hline
\end{tabular}

${ }^{1}$ PC: peso de cacho $(\mathrm{kg})$; PP: peso de penca por cacho (kg); NPC: número de penca por cacho; PMP: peso médio de penca $(\mathrm{kg})$; NBC: número de bananas por cacho.

ns $\mathrm{e} *$ Não-significativo e significativo a $5 \%$ de probabilidade, respectivamente. 
Logicamente que a magnitude da resposta está diretamente relacionada com o nível do nutriente no solo. Para Martin-Prével (1964), em uma plantação de alta produtividade, o nível do K no solo, na instalação do bananal, deve ser, no mínimo, de $8,0 \mathrm{mmol}_{\mathrm{c}} \mathrm{dm}^{-3}$. Entretanto, Malavolta \& Vitti (1984) citam que 4,0 $\mathrm{mmol}_{\mathrm{c}} \mathrm{dm}^{-3}$ correspondem ao valor crítico de $\mathrm{K}$,
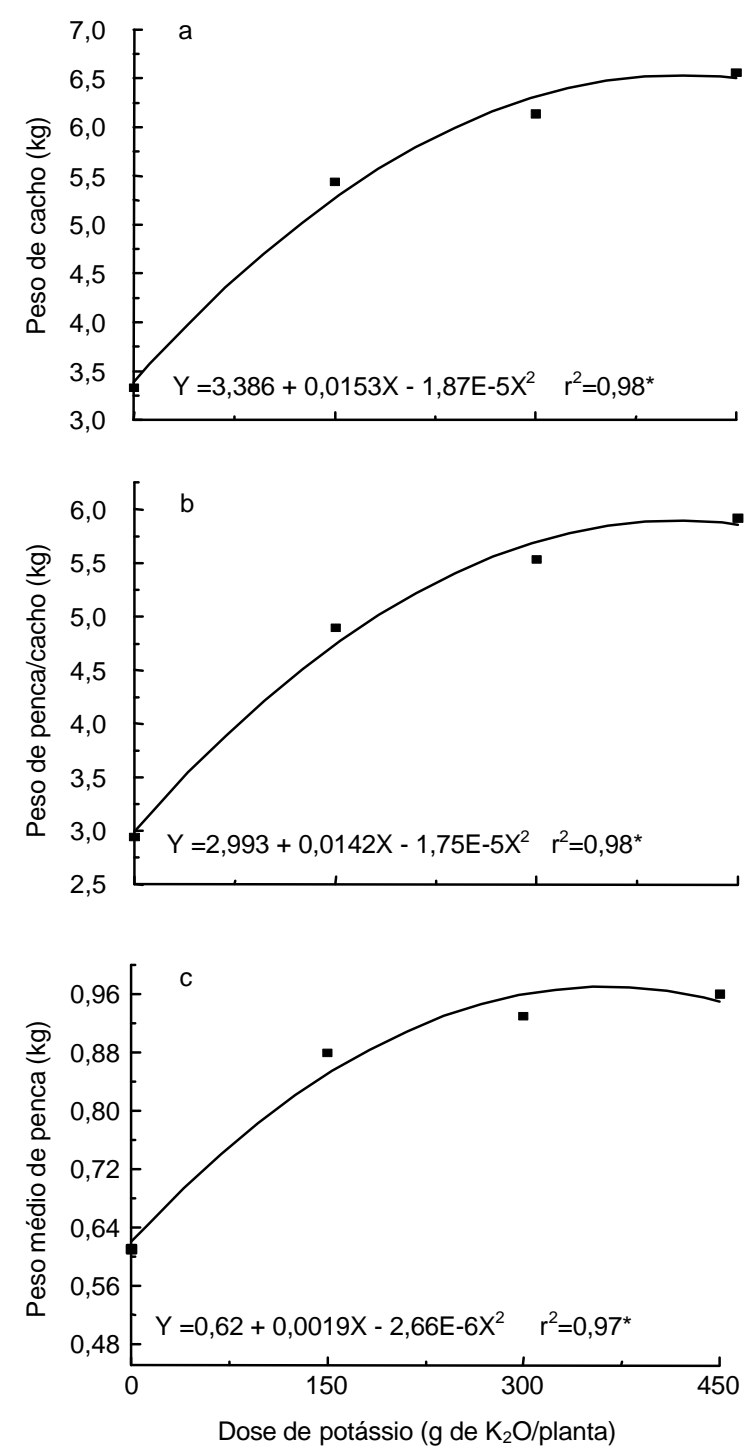

FIG. 2. Efeito de doses de K sobre o peso de cacho (a), peso de penca por cacho (b) e peso médio de penca (c) em bananeira, cv. Pioneira, no segundo ciclo produtivo. que possibilita uma previsão de resposta de $81 \%$ da bananeira à adubação potássica.

$\mathrm{O}$ efeito do $\mathrm{K}$ sobre o número de pencas por cacho e de banana por cacho foi menos acentuado que o verificado em relação às outras variáveis de produção. Com base nos modelos de regressão ajustados para essas variáveis (Fig. 3), verificou-se que, com a aplicação da dose máxima de K (450 g/planta de $\mathrm{K}_{2} \mathrm{O}$ ), foram alcançados aumentos de $26 \%$ e $37 \%$, respectivamente, no número de penca por cacho e de banana por cacho, em relação à ausência de adubação.

Com relação ao efeito do $\mathrm{N}$, a análise de regressão indicou que a adição do nutriente promoveu aumento linear sobre o peso de cacho, peso de penca por cacho (Fig. 4), número de pencas por cacho e número de bananas por cacho (Fig. 5). Os resultados ajustados pelas equações de regressão demonstraram
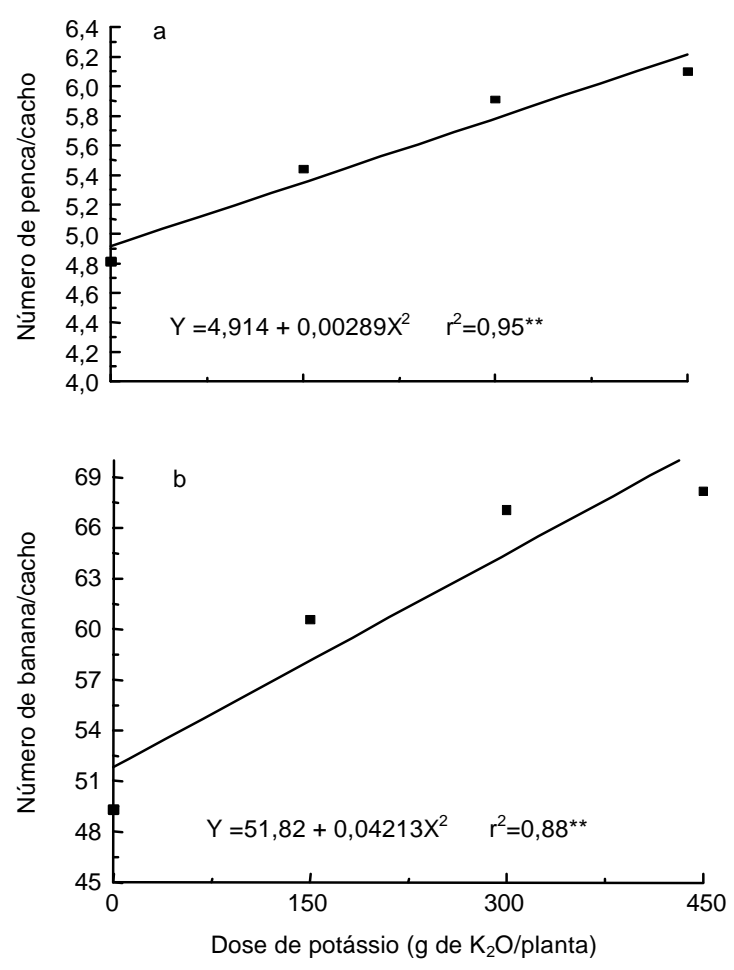

FIG. 3. Efeito de doses de $K$ sobre o número de pencas por cacho (a) e número de bananas por cacho (b) em bananeira, cv. Pioneira, no segundo ciclo produtivo. 
que a aplicação da dose máxima de N (240 g/planta de $\mathrm{N}$ ) proporcionou aumentos expressivos no peso de cacho e de penca por cacho, com estimativas de incremento de produção da ordem de $32 \%$ e $30 \%$, respectivamente, em relação à ausência de aplicação do nutriente. Com base nesses valores, a dose de máxima eficiência física esteve associada a 6,10 e $5,47 \mathrm{~kg}$, respectivamente, quanto às mesmas variáveis. A dose máxima de $\mathrm{N}$ requerida para uma ótima produção de frutos de bananeira satisfaz a recomendação de Soto (1992), que indica a aplicação de $336 \mathrm{~kg} / \mathrm{ha} /$ ano de N.

De modo semelhante ao que ocorreu com o K, a adição de $\mathrm{N}$ sobre o número de pencas por cacho e o número de bananas por cacho favoreceu estimativas de aumento da ordem de $15 \%$ e $23 \%$, valores com menor expressão que os demais.
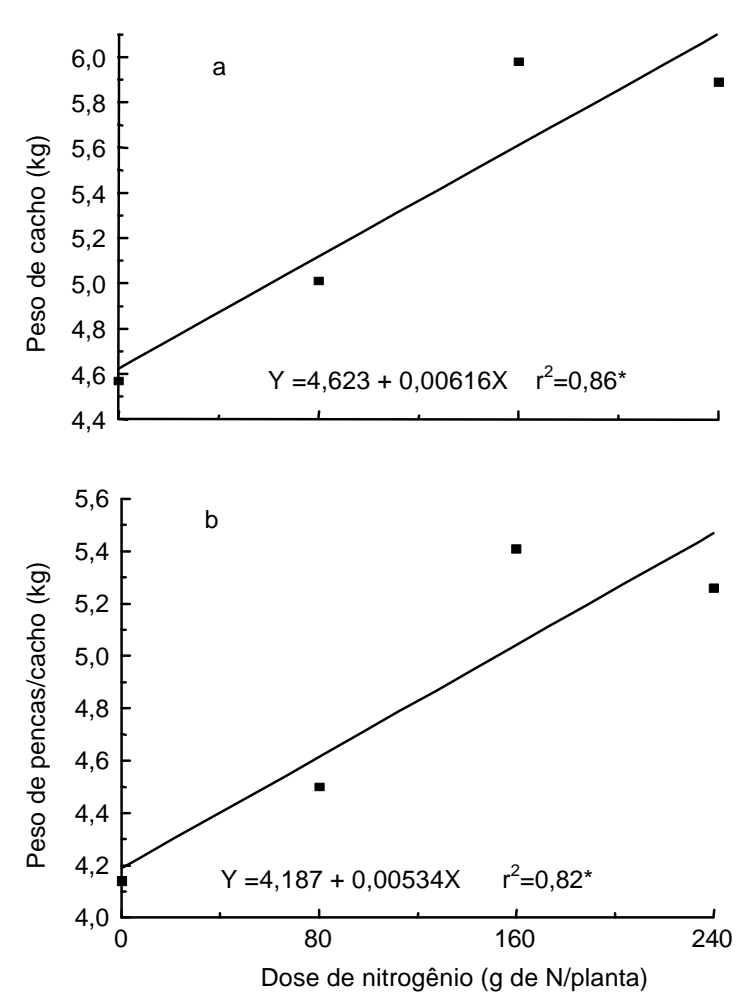

FIG. 4. Efeito de doses de $\mathbf{N}$ sobre o peso de cacho (a) e peso de penca por cacho (b) em bananeira, cv. Pioneira, no segundo ciclo produtivo.
Os resultados do terceiro ciclo de produção da bananeira, demonstraram, por meio da análise de variância (teste $F$ ), que houve efeito significativo apenas em peso de cacho, peso de penca por cacho e peso médio de penca em função da aplicação de doses de K (Tabela 2). Não houve efeito da adubação nitrogenada nem da interação NxK sobre as variáveis estudadas.

De acordo com a análise de regressão, o modelo quadrático foi o que melhor se ajustou aos dados de produção da bananeira, em função da adição de doses de K (Fig. 6). Conforme os modelos de regressão, verificou-se que, para a obtenção da estimativa de produção ótima, em termos de peso de cacho, peso de penca por cacho e peso médio de penca, seria necessária a aplicação de 162, 158 e 118 g/planta de $\mathrm{K}_{2} \mathrm{O}$, respectivamente. Com base nessas estimativas
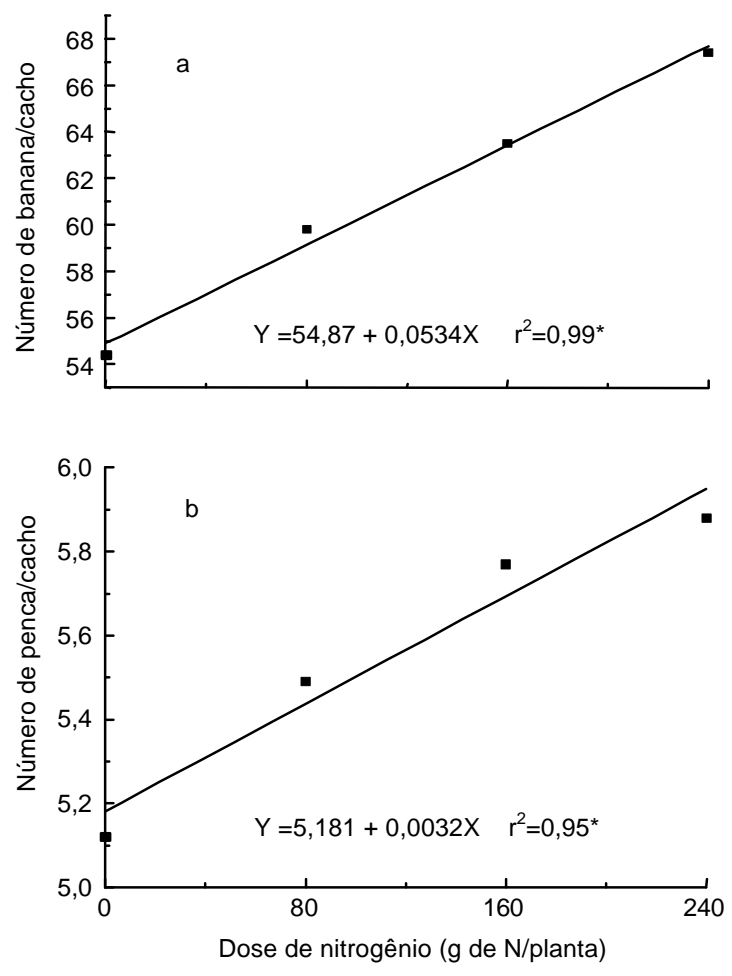

FIG. 5. Efeito de doses de $\mathbf{N}$ sobre o número de bananas por cacho (a) e número de pencas por cacho (b) em bananeira, cv. Pioneira, no segundo ciclo produtivo. 

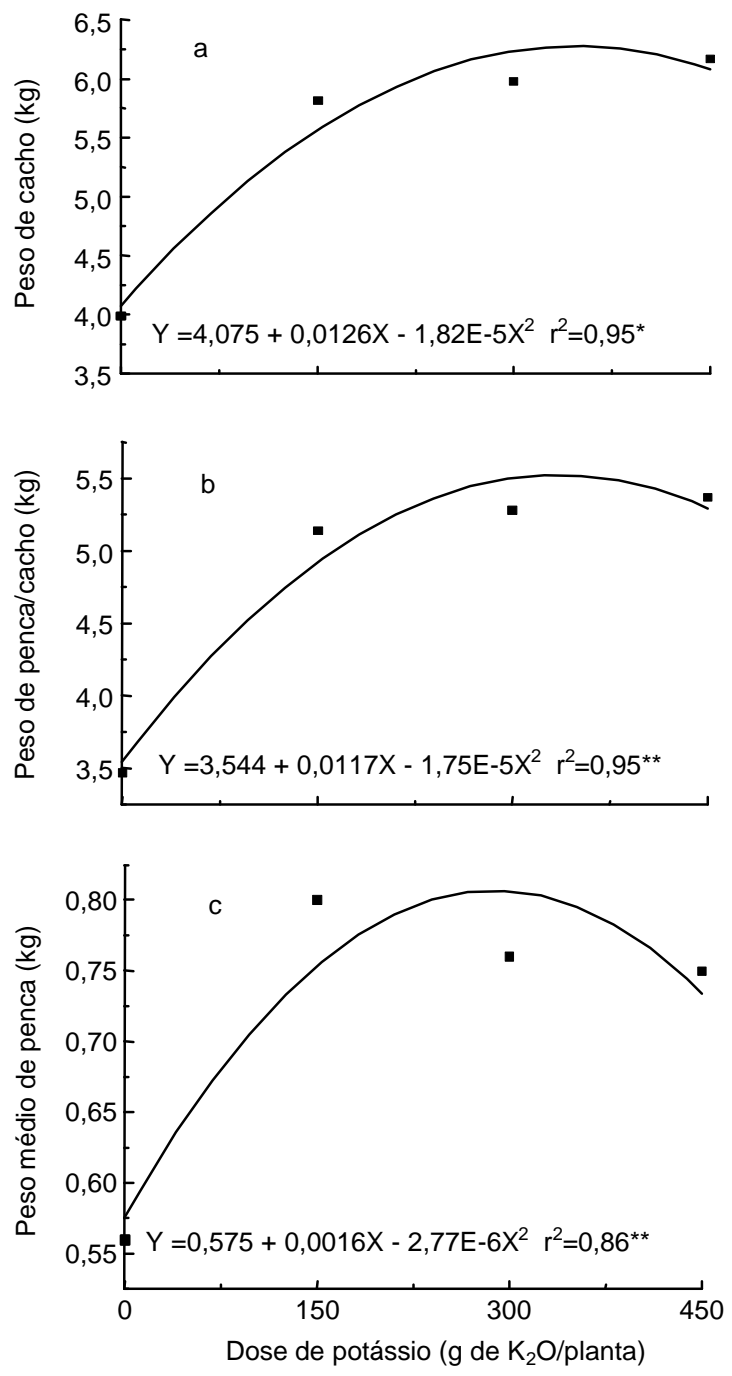

FIG. 6. Efeito de doses de K sobre o peso de cacho (a), peso de penca por cacho (b) e peso médio de penca (c) em bananeira, cv. Pioneira, no terceiro ciclo produtivo.

de produção, observou-se que a adição do nutriente, via adubação mineral, proporcionou aumentos de produção da ordem de $39 \%, 40 \%$ e $26 \%$, respectivamente, nas mesmas variáveis. Tais resultados indicam a importância do K na produção de frutos da bananeira, corroborando com Ho (1969) que obteve boas respostas com a adição de $\mathrm{K}$.

\section{CONCLUSÕES}

1. Durante a fase de crescimento da bananeira, o $\mathrm{N}$ favorece o aumento de altura de planta e de circunferência do pseudocaule.

2. No segundo ciclo produtivo da bananeira a adição do $\mathrm{N}$ promove incrementos em peso de cacho, peso de penca por cacho, número de pencas por cacho e número de bananas por cacho.

3. A aplicação de K durante o segundo e terceiro ciclos de produção da bananeira proporciona aumentos no peso de cacho, peso de penca por cacho e peso médio de penca.

\section{REFERÊNCIAS}

BORGES, A.L.; OLIVEIRA, A.M.G. Nutrição e adubação de bananeira. Brasília : Embrapa-SPI, 1995. 106p. (Publicações Técnicas Frupex, 18).

BORGES, A.L.; OLIVEIRA, A.M.G.; SOUZA, L. da S. Solos, nutrição e adubação da bananeira. Cruz das Almas : Embrapa-CNPMF, 1995. 44 p. (EmbrapaCNPMF. Circular Técnica, 22).

CORAL, R. da S.P. A fruticultura paraense. Belém : Secretaria de Estado de Agricultura, 1998. 18p.

CUNHA, J.F. da; FRAGA JÚNIOR, C. Efeito da adubação mineral, orgânica e calagem na produção de bananeira em várzea litorânea de Caraguatatuba, Estado de São Paulo. Bragantia, Campinas, v.22, n.15, p.159-168, 1963.

EMBRAPA. Serviço Nacional de Levantamento e Conservação de Solos (Rio de Janeiro, RJ). Manual de métodos de análise de solos. Rio de Janeiro, 1979. não paginado.

GALLO, J.R.; BATAGLIA, O.C.; FURLANI, P.R.; HIROCE, R.; FURLANI, A.M.C.; RAMOS, M.T.B.; MOREIRA, R.S. Composição química inorgânica da bananeira (Musa acuminata), cultivar nanicão. Ciência e Cultura, São Paulo, v.24, n.1, p.70-79, 1972.

HEWITT, C.W. Leaf analysis as a guide to the nutrition of bananas. Empire Journal of Experimental Agriculture, London, v.23, n.89, p.11-16, 1955.

HO, C.T. Estudo de correlação entre os rendimentos de frutas e o teor de potássio nas folhas de bananeira. Fertilité, Paris, v.33, p.19-29, 1969. 
MALAVOLTA, E.; VITTI, G.C. Desordens nutricionais e adubação de bananeiras. In: SIMPÓSIO BRASILEIRO SOBRE BANANICULTURA, 1., 1984, Jaboticabal. Anais. Jaboticabal : UNESP/FUNEP, 1984. p.135-158.

MANICA, I.; DEFELIPO, B.V.; CONDÉ, A.R.; LINO, J.; PASSOS, L.C.C. Resposta da bananeira (Musa acuminata) (v. nanicão à adubação com três níveis de nitrogênio, fósforo e potássio. Revista Ceres, Viçosa, v.25, n.142, p.54-95, 1978.

MARTIN-PRÉVEL, P. Os elementos minerais da bananeira e dos seus frutos. Fertilité, Paris, v.22, p.3-14, 1964.

MARTIN-PRÉVEL, P.; MONTAGUT, G. Les interations dans la nutricion minerale bananier. Fruits, Paris, v.21, n.1, p.19-36, 1966.
MOREIRA, R.S. Banana: teoria e prática de cultivo. Campinas : Fundação Cargill, 1987. 335p.

NEVES, R.L.L.; FERREIRA, F.F.H.; MACIEL, R.E.P.; FROTA, J.N.E. Extração de nutrientes em banana (Musa sp.) cv. Pacovan. Ciência Agronômica, Fortaleza, v.22, n.1/2, p.115-120, 1991.

SOTO, M. Bananas: cultivo y comercialización. 2.ed. San José : Litografia y Imprenta, 1992. 520p.

TWYFORD, I.T.; WALMSLEY, D. The mineral composition of the robusta banana plant. III. Uptake and distribution of mineral constituents. Plant and Soil, Dordrecht, v.41, n.3, p.471-491, 1974.

WARNER, R.M.; FOX, R.L. Nitrogen and potassium nutrition of giant cavendish banana in Hawaii. Journal of the American Society for Horticultural Science, Alexandria, v.102, n.6, p.739-743, 1977. 